\title{
Feature Extraction Techniques and Classification Algorithms for EEG Signals to detect Human Stress - A Review
}

\author{
Chetan Umale \\ MIT College of \\ Engineering, \\ Pune, India
}

\author{
Amit Vaidya \\ MIT College of \\ Engineering, \\ Pune, India
}

\author{
Shubham Shirude \\ MIT College of \\ Engineering, \\ Pune, India
}

\author{
Akshay Raut \\ MIT College of \\ Engineering, \\ Pune, India
}

\begin{abstract}
EEG (Electroencephalogram) signal is a neuro signal which is generated due the different electrical activities in the brain. Different types of electrical activities correspond to different states of the brain. Every physical activity of a person is due to some activity in the brain which in turn generates an electrical signal. These signals can be captured and processed to get the useful information that can be used in early detection of some mental diseases. This paper focus on the usefulness of EGG signal in detecting the human stress levels. It also includes the comparison of various preprocessing algorithms ( DCT and DWT.) and various classification algorithms (LDA, Naive Bayes and ANN.). The paper proposes a system which will process the EEG signal and by applying the combination of classifiers, will detect the human stress levels.
\end{abstract}

Keywords: Stress, DWT, KNN, LDA, Naïve Bayes, EEG signal, NeuroSky Mindwave.

\section{INTRODUCTION}

Stress is generally defined as a response of a person to the environmental demands or pressures. It results from interaction between a person and his/her environment that are perceived as straining or exceeding their adaptive capacities and threatening their well-being. It can be understood from above definition that stress is part and parcel of today's life style. If ignored, stress can lead to chronic diseases. The risk factors for stress related diseases are a mixture of personal, interpersonal and social variables. Thus, it can affect various phases of our life. So it is necessary to detect stress at an early stage and take appropriate measures.

Now, the question is, Is it possible to detect stress at early stages? Yes, it is. This is done by many psychologists or counselors. But it requires active participation from the person seeking counseling. This might not be possible in some cases when a stressed person is unable to express himself frankly. It makes the job of a counselor difficult. This problem can be solved, if the brain signals are recorded and analyzed to detect stress.

Brain signals are neuron signals. The electrical activity of the neurons inside the brain cause electric potential to be generated across different parts of the brain. The difference between these electric potential levels can be captured and used for various applications including stress detection. These brain signals are called as EEG signals Electroencephalogram signal. Different types of states of the brain are due to different types of electrical activities of brain neurons. So, different signal values correspond to different mental states.
These signals can be captured using various available equipments which generally consists of electrodes which are placed on the scalp with a conductive gel between the electrodes and the scalp. Electrodes are placed at different positions on the scalp which capture the signals from different parts of the brain. Raw EEG signals cannot be used directly for stress detection. Pre-processing is required to extract useful features which can further used with various machine learning algorithms.

The aim of the paper is to review various feature extraction techniques and classification algorithms which can be used for detection of stress levels. Based on the review, a system is proposed which will use a single electrode EEG headset(Neurosky MindWave) to record raw EEG signals which will be pre-processed using Discrete Wavelet Transform(DWT) and classified using a combination of classifiers approach to detect stress levels. Section 1 gives an introduction on how EEG signals can be used in detection of stress. Section 2 contains literature survey. Section 3 contains the proposed system for stress detection. Section 4 is conclusion and Section 5 includes future scope.

\section{LITERATURE SURVEY}

\subsection{EEG}

Electroencephalography (EEG) is nothing but recorded electrical activity generated by brain [2].The first report on electrical brain activity in humans was published in 1929 which allowed doctors and scientists to observe the brain in action in a meaningful way [5]. There are millions of neurons in our brain. These activities generate millions of small electric voltage fields. The aggregate of these voltage fields can be detected by electrodes placed on the scalp. Thus we can say that, EEG is the superposition of many smaller signals. The 
amplitude of these signals ranges from $1 \mu \mathrm{V}$ to $100 \mu \mathrm{V}$ in a normal person.

The different electrical frequencies in EEG can be associated with different physical actions and mental states [3]. So EEG shows a wide variations in amplitude depending on external stimulation and different internal mental states. The different frequency bands are Delta, theta, alpha, beta and gamma. Frequency bands associated with the different mental states are given in the Table 1.

Table 1: EEG frequency bands

\begin{tabular}{|c|c|c|}
\hline $\begin{array}{c}\text { Brainwave } \\
\text { type }\end{array}$ & $\begin{array}{c}\text { Frequency } \\
\text { range(Hz) }\end{array}$ & Mental states and conditions \\
\hline Delta & 0.1 to 3 & $\begin{array}{c}\text { Deep, dreamless sleep, } \\
\text { unconscious }\end{array}$ \\
\hline Theta & 4 to 7 & $\begin{array}{c}\text { Intuitive, creative, recall, } \\
\text { fantasy }\end{array}$ \\
\hline Alpha & 8 to 12 & $\begin{array}{c}\text { Relaxed but not drowsy, } \\
\text { tranquil }\end{array}$ \\
\hline Low Beta & 12 to 15 & $\begin{array}{c}\text { Formerly SMR, relaxed yet } \\
\text { focused }\end{array}$ \\
\hline $\begin{array}{c}\text { Midrange } \\
\text { Beta }\end{array}$ & 16 to 20 & $\begin{array}{c}\text { Thinking, aware of self \& } \\
\text { surrounding }\end{array}$ \\
\hline High Beta & 21 to 30 & Alertness, agitation \\
\hline Gamma & 30 to 100 & $\begin{array}{c}\text { Motor functions, higher mental } \\
\text { activity }\end{array}$ \\
\hline
\end{tabular}

\subsection{SIGNAL ACQUIRING METHODS}

Various methods are used for acquiring EEG signals. They differ in the way the electrodes are placed. The methods can be categorized as follows:

a) Invasive: Invasive EEG recordings are those recordings that are captured with electrodes that are placed on the surface or within the depth of the brain[6]. These type of methods are generally used in medical surgeries or implants. Again the two types of electrodes used in this method are

i) Subdural EEG electrodes: Subdural EEG electrodes are the electrodes which sit over the surface of the brain. The placement of these electrodes is often confirmed with coregistration on an MRI scan image.

ii) Depth EEG electrodes: Depth EEG electrodes are those which are placed within the substance of the brain.

b) Non-invasive: Non-invasive EEG recordings are those that are captured with electrodes that are placed on the scalp rather than placing it on the surface or within the depth of the brain. Electrodes used here are small metal discs which are made of stainless steel, tin, gold or silver covered with a silver chloride coating. They are placed on the scalp in special positions. These positions are specified using the International 10/20 system. Each electrode site is labeled with a letter and a number. The letter refers to the area of brain underlying the electrode e.g. FFrontal lobe and T - Temporal lobe. Even numbers denote the right side of the head and odd numbers the left side of the head
[7]. A mapping of these electrode positions according to the 1020 international system is shown in the Figure 1.

This system is used for multichannel electrode system which are generally used for research purpose and are complex and not portable. Alternative to such systems is single electrode system which uses single channel for recording signal and are simpler and portable. An example of such system is NeuroSky MindWave headset. The comparison between the signals captured by single channel NeuroSky MindWave and multichannel Biopac system is. The red line is Biopac and a

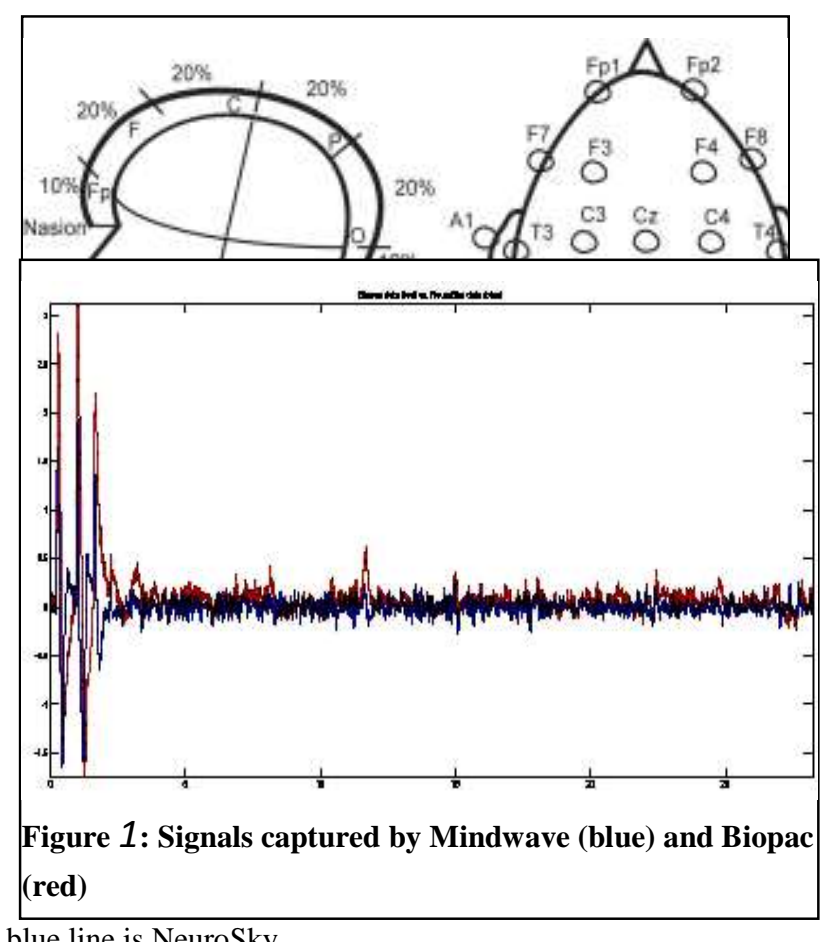

blue line is NeuroSky.

\subsection{MATLAB}

MATLAB (Matrix Laboratory) is a multi-paradigm numerical computing environment and high level programming language developed by Math works. MATLAB allows matrix manipulations, plotting of functions and data, implementation of algorithms, creation of user interfaces, and interfacing with programs written in other languages, including $\mathrm{C}, \mathrm{C}++$, Java, Fortran and Python[9].EEG signals being electrical signals are vulnerable to outside interference and artifacts. Using signal processing capabilities of MATLAB, these problems can be resolved effectively. MATLAB provides an interactive toolbox called EEGLAB, which is effective for continuous and eventrelated EEG signals analysis using independent component analysis (ICA), time/frequency analysis (TFA), as well as standard averaging methods [10]. EEGLAB supports loading of existing EEG datasets as well as real time EEG datasets through software like Neuroscan. 


\subsection{Pre-processing}

EEG signals are non-stationary and non-linear. EEG signals are susceptible to noise and interference caused by eye movement and muscle movement. The electronic devices in the vicinity can also cause interference. Also, the amount of raw data required for classification is impractical for most machine learning algorithms. Thus feature extraction is necessary for successful classification. Pre-processing includes transformation of EEG signals from time domain to frequency domain and removal of noise and artifacts.

A variety of feature extraction methods exist for BCI applications, such as Discrete Cosine Transform (DCT), Discrete Wavelet Transform (DWT).

\subsubsection{Discrete Cosine Transform (DCT)}

Discrete Cosine Transform is a method to convert time series signals into frequency components. In context of BCI, DCT is used to calculate maximum, minimum and mean value of EEG signal. The one-dimensional DCT for a list of $\mathrm{N}$ real numbers is expressed by the following formula:

$$
\begin{gathered}
Y(u)=\sqrt{\frac{2}{N} a(u) \sum_{\mathrm{x}=0}^{N-1} f(x) \cos \left(\frac{\pi(2 \mathrm{x}+1) \cdot u}{2 N}\right)} \\
\text { where } \\
a(j)=\frac{1}{\sqrt{2}} \text { if } \mathrm{j}=0 \\
a(j)=1 \text { if } j \neq 0
\end{gathered}
$$

The input is a set of $\mathrm{N}$ data values (EEG samples) and the output is a set of N DCT transform coefficients $Y(u)$. The first coefficient $\mathrm{Y}(0)$ is called the DC coefficient and it holds average signal value.. The rest coefficients are referred to as the AC coefficients [11]. DCT produces concentrated signals, where the energy is concentrated into few coefficients. Thus DCT is effective for data compression which leads to reduced size of input vector for machine learning algorithms and also reduces time required for learning.

\subsubsection{Discrete Wavelet Transform (DWT)}

Wavelet transform is the process of expressing any general function as an infinite series of wavelets. The main idea behind wavelet analysis is of expressing a signal as a linear combination of the particular set of functions by shifting and expanding the original wavelet (mother wavelet). This decomposition gives a set of coefficients called as wavelet coefficients, due to this the signal can be reconstructed as a linear combination of the wavelet functions weighted by the wavelet coefficients. The main feature of the wavelets is that most of their energy is restricted to a finite time interval .This is called as time-frequency localization. Frequency localization means that the Fourier transform is band limited. This timefrequency localization provides good frequency localization at low frequencies and good time localization at high frequencies. This produces segmentation of the time-frequency plane that is appropriate for most physical signals, especially those of a transient nature. This transform when applied to EEG signal will reveal features that are transient in nature [8].

Discrete wavelet transform depends on low pass filter $g$ and high pass filter $h$. The working is based on two important functions namely wavelet function $\varphi_{\mathrm{i}, \mathrm{l}}(\mathrm{k})$ and scale function $\psi_{\mathrm{i}, 1}(\mathrm{k})$ which can be defined as :

$$
\begin{aligned}
& \varphi_{\mathrm{i}, 1}(\mathrm{k})=2^{\mathrm{i} / 2} \mathrm{~g}_{\mathrm{i}}\left(\mathrm{k}-2^{\mathrm{i} l}\right) \\
& \psi_{\mathrm{i}, \mathrm{l}}(\mathrm{k})=2^{\mathrm{i} / 2} \mathrm{~h}_{\mathrm{i}}\left(\mathrm{k}-2^{\mathrm{i} l}\right)
\end{aligned}
$$

where the factor $2^{\mathrm{i} / 2}$ is an inner product normalization, $i$ and 1 are the scale parameter and the translation parameter, respectively. The DWT decomposition is described as:

$$
\begin{aligned}
& \mathrm{a}_{\mathrm{i})}(\mathrm{l})=\mathrm{x}(\mathrm{k})^{*} \varphi_{\mathrm{i}, \mathrm{l}}(\mathrm{k}) \\
& \mathrm{d}_{(\mathrm{i})}(\mathrm{l})=\mathrm{x}(\mathrm{k})^{*} \psi_{\mathrm{i}, \mathrm{l}}(\mathrm{k})
\end{aligned}
$$

where $a_{(i)}(1)$ is the approximation coefficient and $d_{(i)}(1)$ is the detail coefficient at resolution $i$.

The DWT decomposition of the input signal into different frequency bands is obtained by consecutive high-pass and lowpass filtering of the time domain signal. This decomposition is shown in the Figure 3.

In the given diagram, $x[n]$ is the mother wavelet, $h[n]$ is the high pass filter and $\mathrm{g}[\mathrm{n}]$ is the low pass filter. EEG signals do not have any useful frequency components above $30 \mathrm{~Hz}$ [8].So the decomposition levels can be selected as 5. So the final relevant wavelet decomposition will be obtained at level A5.Sample EEG signal decomposition is shown in figure 4 . 


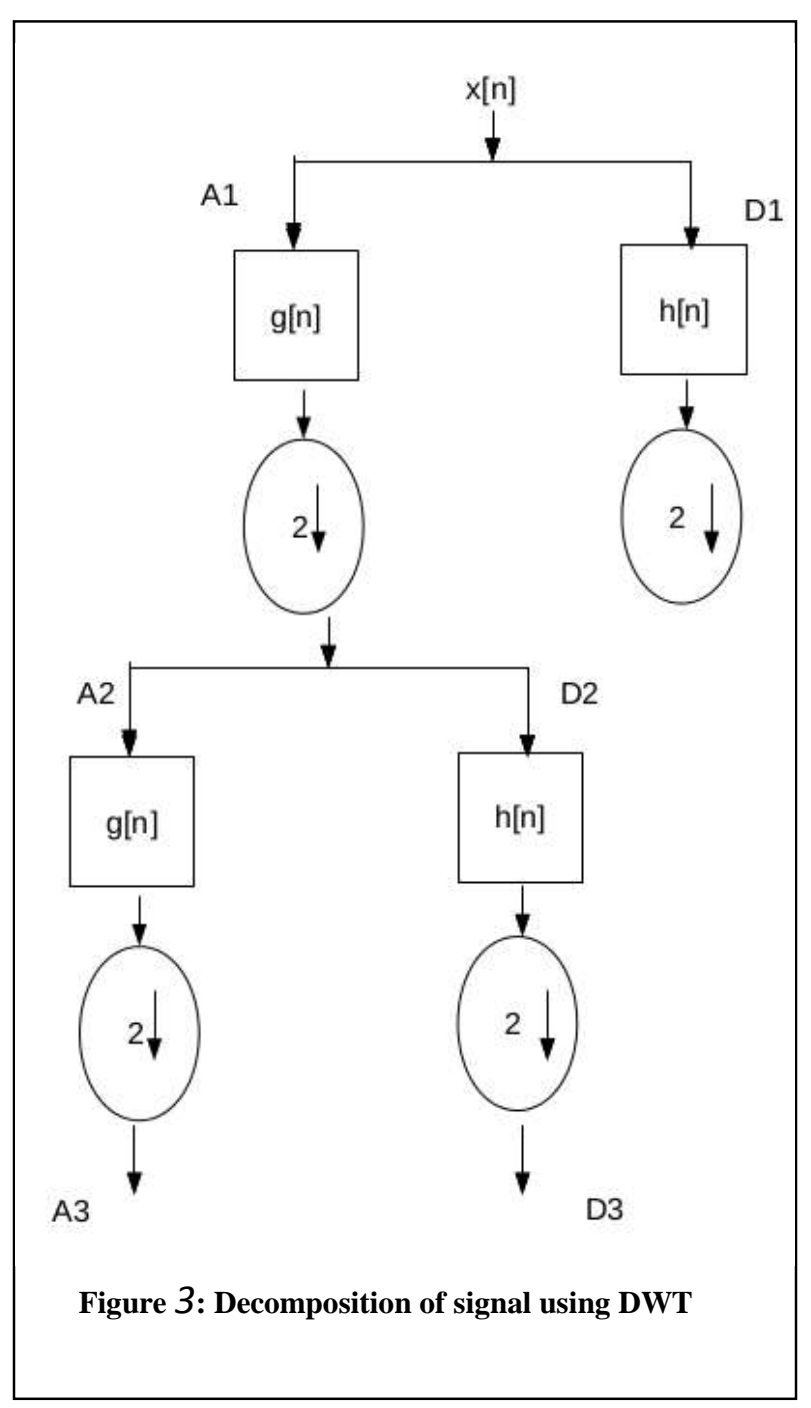

\subsection{CLASSIFICATION}

\subsubsection{K-Nearest Neighbor $(\mathrm{KNN})$}

$\mathrm{K}$-nearest neighbor is an instance-based, lazy and supervised learning algorithm. $\mathrm{KNN}$ is a simple algorithm that stores all available cases and classifies new cases based on a similarity measure. $\mathrm{KNN}$ is a non-parametric method that classifies the data by comparing the training data and testing data based on estimating the feature values[12]. These feature values are calculated by using the distance function such as Euclidean distance which is not difficult if the given parameter values are numeric. An object is then classified by the majority vote of its neighbors, with the object being assigned to the class most common among its $\mathrm{k}$ nearest neighbors. The value of the $\mathrm{k}$ refers to how many nearest values should be considered before the output class is decided.

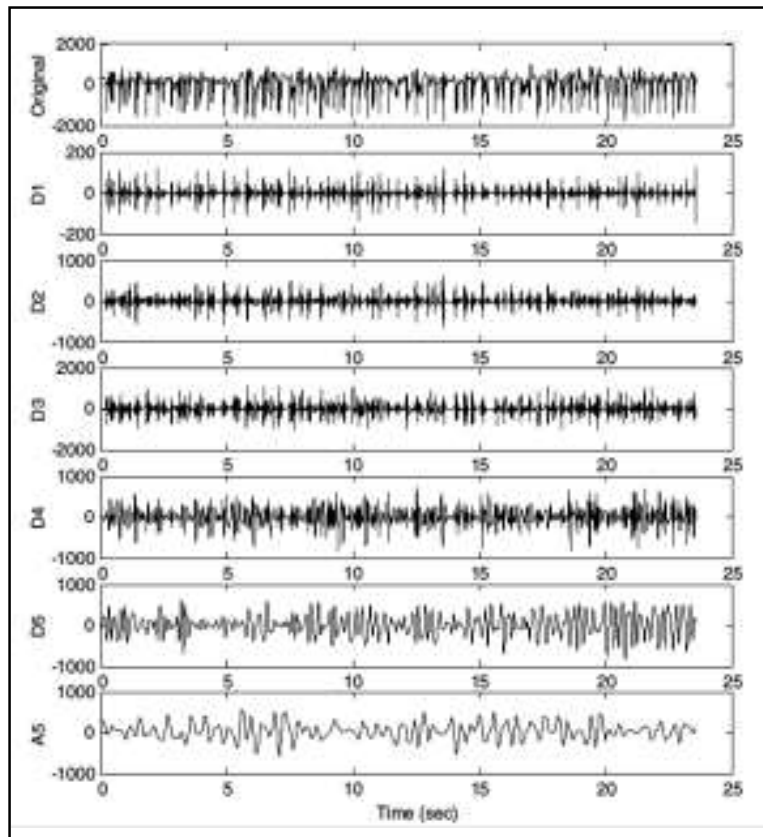

Figure 4: Decomposition of EEG signal using DWT

A commonly used distance metric is the Euclidean distance. The Euclidean distance of two points or tuples, say, $\mathrm{X}_{1}=\left(\mathrm{x}_{11}, \mathrm{x}_{12}, \ldots \ldots, \mathrm{x}_{1 \mathrm{n}}\right)$ and $\mathrm{X}_{2}=\left(\mathrm{x}_{21}, \mathrm{x}_{22}, \ldots \ldots, \mathrm{x}_{2} \mathrm{n}\right)$, is

$$
\operatorname{dist}\left(X_{1}, X_{2}\right)=\sqrt{\sum_{i=1}^{n}\left(x_{1 i}-x_{2 i}\right)^{2}}
$$

where, $\mathrm{x}_{1 \mathrm{i}}$ and $\mathrm{x}_{2 \mathrm{i}}$ represents the training and testing data respectively[13]. Different attributes are measured on different scales, so if the Euclidean distance formula is used directly, the effect of some attributes might be completely dwarfed by others that have larger scales of measurement.

After feature extraction process the EEG training data and test data is passed to the classification process. Then Euclidean distance is calculated between each EEG training sample and testing sample. The class for first $\mathrm{K}$ neighbors is considered and the majority vote is the classified class. The accuracy for the KNN is high as compared to the other classifiers.

\subsubsection{Linear Discriminant Analysis (LDA)}

Linear discriminant analysis (LDA) is one of the most popular classification algorithms for Brain Computer Interface applications, and has been used successfully in a large number of systems. LDA linearly transforms data from high dimensional space to low dimensional space. Finally, the decision is made in the low dimensional space. Thus the definition of the decision boundary plays an important role in classification process. During this process, the class distributions having some finite variance will be still kept in the projected space. Hence, we assume that if the mean and variance of the projected data is considered for the calculation of the decision boundary, it may extend LDA method to deal 
with the practical heteroscedastic distribution data, which derives Z-LDA.

Consider the case of two classes (x11, x12, x13, ... $\mathrm{x} 1 \mathrm{~m}) € \mathrm{C} 1$ and $(\mathrm{x} 21, \mathrm{x} 22, \mathrm{x} 23$, $\mathrm{x} 2 \mathrm{n})$ and $n$ being number of training samples.

$\mathrm{X}=(\mathrm{x} 11, \mathrm{x} 12, \mathrm{x} 13$, , $1 \mathrm{~m}, \mathrm{x} 21, \mathrm{x} 22, \mathrm{x} 23$ $x 2 n)$

be our input sample.

Calculate weight sum $\mathrm{y}(\mathrm{X})$ by, $\mathrm{y}(\mathrm{X})$

$$
y(X)=W^{T} \not x
$$

where WT is weight vector.

Now, the parameters related to Gaussian distribution mean $(\mu)$ and standard deviation $(\sigma)$ are calculated as :-

$$
\begin{aligned}
\mu_{1} & =\frac{1}{m} \sum_{\mathbf{x} \in C_{1}} y(\mathbf{x}) \\
\mu_{2} & =\frac{1}{n} \sum_{\mathbf{x} \in C_{2}} y(\mathbf{x}) \\
\sigma_{1} & =\sqrt{\frac{1}{m} \sum_{\mathbf{x} \in C_{1}}\left(y(\mathbf{x})-\mu_{1}\right)^{2}} \\
\sigma_{2} & =\sqrt{\frac{1}{n} \sum_{\mathbf{x} \in C_{2}}\left(y(\mathbf{x})-\mu_{2}\right)^{2}}
\end{aligned}
$$

where $\mu 1, \mu 2$ and $\sigma 1, \sigma 2$ and mean and standard deviations of two training set samples $\mathrm{C}_{\mathrm{K}}(\mathrm{K}=1,2)$.

During classification process, when any sample $\mathrm{X}$ is input, first calculate weight sum $\mathrm{y}(\mathrm{x})$ and then perform following normalization procedure:-

$\mathrm{z} 1=(\mathrm{y}(\mathrm{x})-\mu 1) / \mathrm{ro} 1$

$\mathrm{z} 2=(\mathrm{y}(\mathrm{x})-\mu 2) / \mathrm{ro} 2$

where $\mathrm{z} 1$ and $\mathrm{z} 2$ are $\mathrm{z}$-scores to calculate how much weight sums of given input sample is close to the training samples. Finally, the larger value among these $\mathrm{z}$ scores gives the final classification class for the input. That means, if $z 1>z 2$, the sample is going to be classified in class $\mathrm{c} 1$; otherwise $\mathrm{c} 2$.

\subsubsection{Naive Bayes}

Probability can be interpreted from two views: Objective and Subjective. The Subjective probability is called as Bayesian Probability. Bayesian Probability is the process for using the probability for predicting the likelihood of certain events occurring in the future. Naive Bayes is a conditional probability model where Bayes' theorem is used to infer the probability of hypothesis under the observed data or evidence [14]. Bayes theorem states that

$$
\text { posterior }=\frac{\text { prior } * \text { likelihood }}{\text { evidence }}
$$

$$
p(B \mid A)=\frac{p(A \mid B) p(B)}{p(A)}
$$

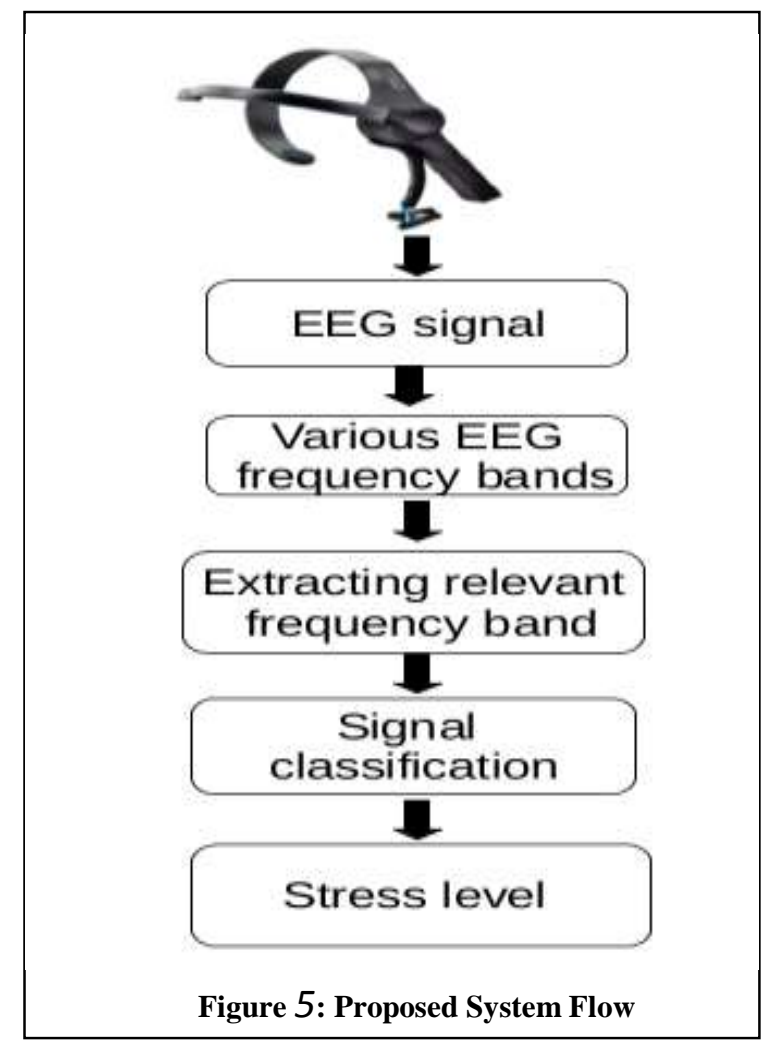

When dealing with continuous data generated from EEG signals, a typical assumption is that the continuous values associated with each class are distributed according to a Gaussian distribution. First the data is segment by the class, and then compute the mean and variance of $\mathrm{x}$ in each class. Let $\mu_{\mathrm{c}}$ be the mean of the values in $\mathrm{x}$ associated with class $c$, and let $\sigma_{c}{ }^{2}$ be the variance of the values in $\mathrm{x}$ associated with class $c$. Then, the probability distribution of some value given a class, $\mathrm{p}(\mathrm{x}=\mathrm{v} \mid \mathrm{c})$, can be computed by plugging $\mathrm{v}$ into the equation for a Normal distribution parameterized by $\mu_{\mathrm{c}}$ and $\sigma_{\mathrm{c}}{ }^{2}$. That equation is :

$$
f(x)=\frac{1}{\sigma \sqrt{2 \pi}} e^{-\frac{(v-\mu)^{2}}{2 \sigma^{2}}}
$$




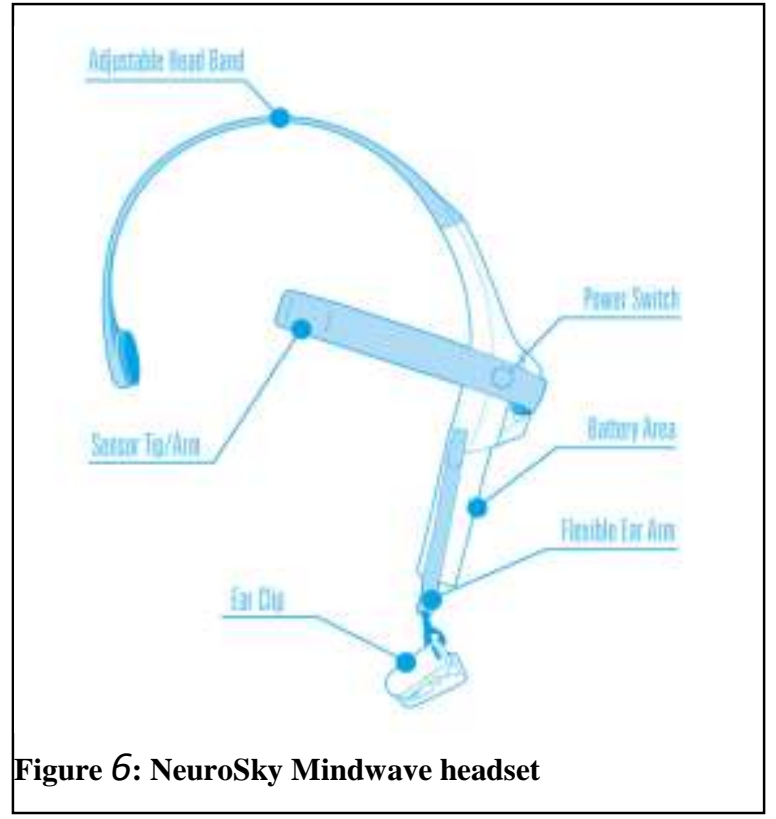

\section{PROPOSED SYSTEM3.1 Data}

\section{collection equipment Here the data required}

is the EEG signal. In the proposed system, the equipment to be used in the data collection is NeuroSky Mindwave headset which uses single electrode for capturing the EEG signal. There are various noise sources in this process such as muscle movement or any electrical device in the vicinity. Primary filtering of these noise signals is done by the ear clip which acts as reference or ground.
The captured signal contains various EEG frequency bands. The frequency range relevant for stress level detection is 4 to $40 \mathrm{~Hz}$ [2]. Thus, only these frequencies are extracted. This process is called feature extraction. In the proposed system, DWT is used to preprocess the data as it has the unique advantage of time-frequency localization [8].

Once the preprocessing is done, next task is to classify the signal into appropriate stress level. When a single classifier is used, it is difficult to identify the misclassification error. The solution to this problem is to develop a more complex classifier with a little misclassification rate. This approach may seem promising but it will make the system complex. Another approach is to use combination of simple classifiers rather than using one complex classifier. One more advantage of this approach is that even if one of the classifier misclassifies the data, the other classifiers can rectify this error.

In the proposed system, an approach of combination of classifiers is used. The classifiers which are to be uses are KNN, Naive Bayes and LDA. The output class will be decided by voting and the class which gets majority of votes will be the output class.

\section{CONCLUSION}

Early detection of stress can help in prevention of chronic mental illness. Recording and analyzing EEG signals can be an effective tool for this purpose. Different feature extraction techniques and classification algorithms for EEG signal analysis are discussed in this paper. This review suggests use of single channel EEG headset which provides a portable and affordable alternative to traditional multichannel equipments.

\section{FUTURE WORK}

The paper proposed the methodology for detecting human stress level in real time. As mentioned, early detection and treatment of stress is important. Music therapy is a good option for stress treatment. Music with appropriate frequency can be played automatically corresponding to detected stress level. Another future application of EEG signal can be a biometric authentication system, as pattern of EEG signal captured from every person is unique.
Figure 6 shows the general flow of the proposed system. EEG signal is captured using the NeuroSky Mindwave. Processing is done in the MATLAB environment using the EEGLAB interface. EEGLAB provides functions to capture data in numerous scenarios such as at different sampling rate.

\subsection{System flow}




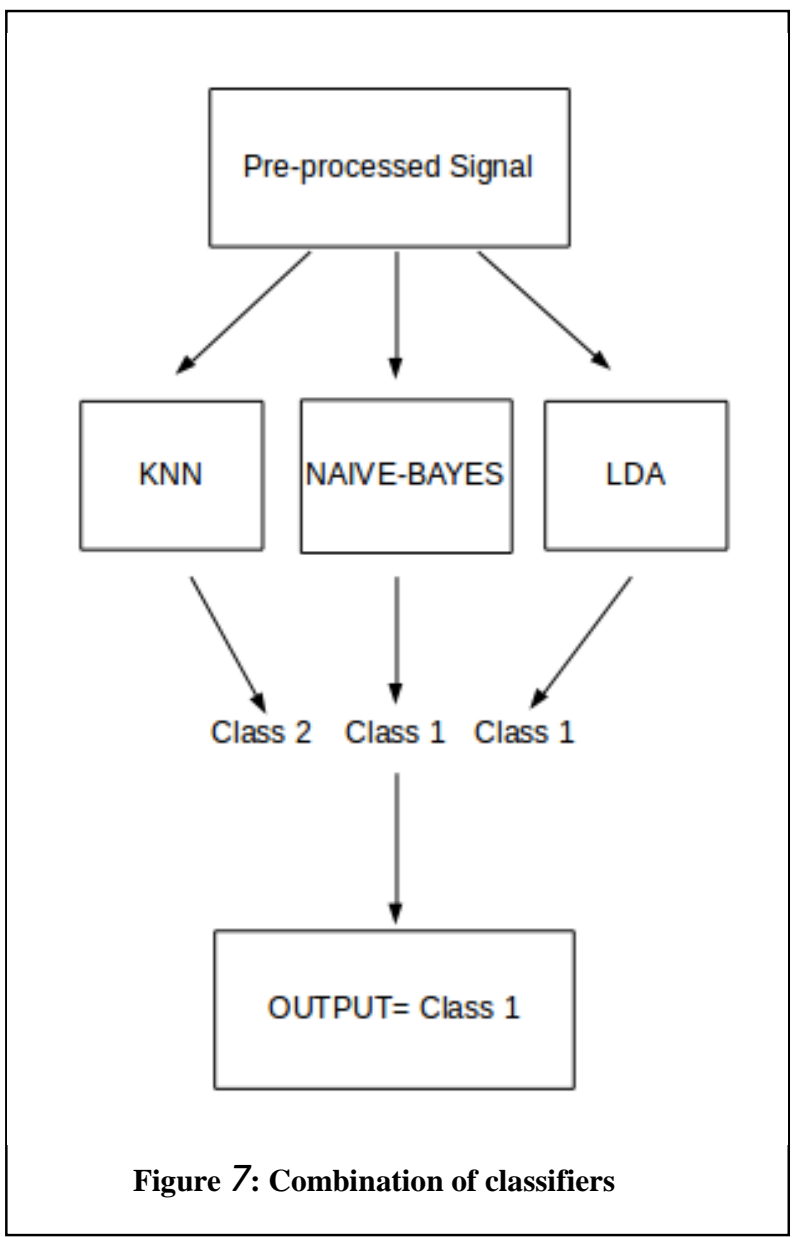

\section{REFERENCES}

[1] http://www.stress.org.uk/what-is-stress.aspx

[2] NeuroSky Inc. Brain Wave Signal (EEG) of NeuroSky, Inc.(December 15, 2009). [Online]. Available:http://frontiernerds.com/files/neurosky-vsmedical-eeg.pdf

[3] Erik Andreas Larsen, "Classification of EEG Signals in a Brain-Computer Interface System" ,Norwegian University of Science and Technology Department of Computer and Information Science,June 2011
[4] D. Puthankattil Subha, Paul K. Joseph, Rajendra Acharya U, Choo Min Lim, "EEG Signal Analysis: A Survey" ,J Med Syst (2010) 34:195-212

[5] D. A. Kaiser. What is quantitative EEG. [Online].Available:http://www.skiltopo.com/skil3/whatis-qeeg-by-kaiser.pdf

[6] https://my.clevelandclinic.org/services/neurological_insti tute/epilepsy/diagnostics-testing/invasive-eeg-monitoring

[7] http://www.medicine.mcgill.ca/physio/vlab/biomed_sign als/eeg_n.htm

[8] Abdulhamit Subasi, "EEG signal classification using wavelet feature extraction and a mixture of expert model",Expert Systems with Applications 32 (2007) 1084-1093,2006 Elsevier Ltd.

[9] https://en.wikipedia.org/wiki/MATLAB

[10] http://sccn.ucsd.edu/eeglab/

[11] Darius Birvinskas,Vacius Jusas,Ignas Martišius, Robertas Damaševičius, "Data Compression of EEG Signals for Artificial Neural Network Classification",ISSN 2335884X (online) INFORMATION TECHNOLOGY AND CONTROL, 2013, Vol.42, No.3

[12] Tatiur Rahman, Apu Kumer Ghosh, Md. Maruf Hossain Shuvo, Md. Mostafizur Rahman, "Mental Stress Recognition using K-Nearest Neighbor(KNN) Classifier on EEG Signals", International Conference on Materials, Electronics \& Information Engineering, ICMEIE-2015.

[13] Chee-Keong Alfred Lim and Wai Chong Chia, "Analysis of Single-Electrode EEG Rhythms Using MATLAB to Elicit Correlation with Cognitive Stress", International Journal of Computer Theory and Engineering, Vol. 7, No. 2, April 2015

[14] Juliano Machado Alexandre Balbinot, Adalberto Schuck, "A study of the Naive Bayes classifier for analysing imaginary movement EEG signals using the Periodogram as spectral estimator", Bio-signals and Bio-robotics Conference (BRC), 2013 ISSNIP, IEEE conference publication. 